\title{
Precision phenomenology and differential equations approach to master integrals
}

\author{
Costas G. Papadopoulos, Damiano Tommasini*, Christopher Wever ${ }^{\dagger}$ \\ Institute of Nuclear Physics, NCSR "Demokritos", Athens, Greece \\ E-mail: costas.papadopoulos@cern.ch, tommasini@inp.demokritos.gr, \\ weverainp. demokritos.gr
}

\begin{abstract}
A short review is given of the simplified differential equations approach to Master Integrals, which was recently proposed by one of the authors. We show its applicability by calculating some non-trivial two-loop planar Master Integrals, namely those contributing to amplitudes of massive diboson $V V^{\prime}$ production at the LHC with massless internal lines.
\end{abstract}

Proceedings of the Corfu Summer Institute 2014

3-21 September 2014

Corfu, Greece

\footnotetext{
*Speaker.

† Supported by the Research Funding Program ARISTEIA, HOCTools (co-financed by the European Union (European Social Fund ESF) and Greek national funds through the Operational Program "Education and Lifelong Learning" of the National Strategic Reference Framework (NSRF)).
} 


\section{Introduction}

At the first run of the LHC, proton collisions have reached the record-setting high energies of $8 \mathrm{TeV}$. The second run of the LHC, which is expected to start in 2015, will push the energy and luminosity even higher. In order to keep up with the increasing experimental accuracy as more data is collected, more precise theoretical predictions and higher loop calculations will be required.

With the better understanding of reduction of one-loop amplitudes to a set of Master Integrals (MI) based on unitarity methods $[2,1]$ together with their implementation at the integrand level via the OPP method [3, 4], one-loop calculations have been fully automated in many numerical tools $[5,6,7,8,9,10]$. In recent years, a lot of progress has been made towards the extension of on-shell reduction methods to the two-loop order at the integral $[11,12]$ as well as the integrand $[13$, $14,15]$ level. Contrary to the MI at one-loop, which have been known for a long time already [16], a complete library of MI at two-loops is still missing.

Starting from the works of $[17,18,19]$, there has been a building consensus that the so-called Goncharov Polylogarithms (GPs) form a functional basis for many MI. A very fruitful method for calculating MI and expressing them in terms of GPs is the differential equations (DE) approach [20, 21], which has been used in the past two decades to calculate various MI at two-loops [22, 23, 24, $25,26,27,28,29,30,31,32,33$ ]. In [34] a variant of the traditional DE approach to MI was presented, which was coined the Simplified Differential Equations (SDE) approach. In this paper a short review of the SDE approach and an application to the four-point two-loop planar MI with two different massive external legs and massless internal propagators is presented [35].

\section{Simplified differential equations approach}

The SDE approach was introduced in [34] and its main points are reviewed in this section. Assume one is interested in calculating an $l$-loop Feynman integral whose graph with external incoming momenta $\left\{p_{j}\right\}$ is shown on the left hand side in Figure 1. The method has been developed for massless internal lines and therefore all propagators are massless in the rest of this paper. All relevant Feynman integrals are a subset of the following class of loop integrals:

$$
G_{a_{1} \cdots a_{n}}\left(\left\{p_{j}\right\}, \varepsilon\right)=\int\left(\prod_{r=1}^{l} d^{d} k_{r}\right) \frac{1}{D_{1}^{2 a_{1}}(k, p) \cdots D_{n}^{2 a_{n}}(k, p)}, \quad D_{i}(k, p)=c_{i j} k_{j}+d_{i j} p_{j},
$$

where the denominators are defined in such a way that all scalar product invariants can be written as a linear combination of them. The exponents $a_{i}$ are integers and may be negative in order to generate numerators.

The above class of Feynman integrals satisfy so-called integration by parts (IBP) identities $[36,37]$ in dimensional regularization (DR) with $d=4-2 \varepsilon$ :

$$
\int\left(\prod_{r=1}^{l} d^{d} k_{r}\right) \frac{\partial}{\partial k_{j}^{\mu}}\left(\frac{v^{\mu}}{D_{1}^{2 a_{1}} D_{2}^{2 a_{2}} \cdots D_{n}^{2 a_{n}}}\right) \stackrel{D R}{=} 0
$$

where the vector $v_{\mu}$ is in practice taken to be one of the loop or external momenta. By solving the above identities one can reduce any integral $G_{a_{1} \cdots a_{n}}$ as a linear combination of MI with rational coefficients in the scalar products and space-time dimension $d$. Reduction by IBP is by now implemented in public tools $[39,38]$. 

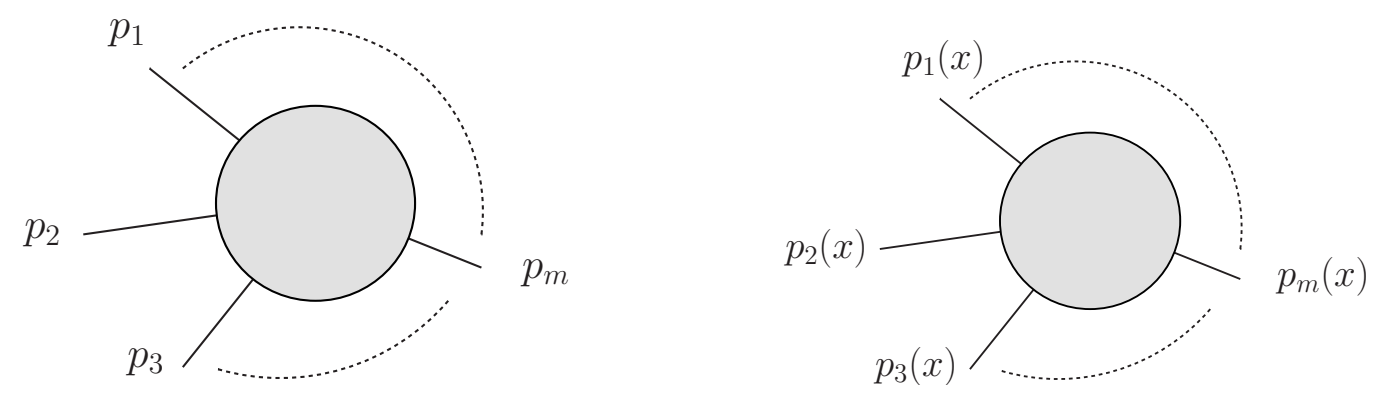

Figure 1: General graphs represented by (2.1) on the left and (2.5) on the right.

If a basis vector of MI $\vec{G}^{M I}(s)=\left\{G_{b_{1} \cdots b_{n}} \mid\left(b_{1} \cdots b_{n}\right) \in\right.$ Master Integrals $\}$ is known, then any integral $G_{a_{1} \cdots a_{n}}$ may be calculated after IBP reduction. In the traditional DE method $\vec{G}^{M I}(s)$ is differentiated with respect to a Lorentz invariant (or set of invariants) $s$ and the resulting integrals are reduced by IBP to give a linear system of DE for $\vec{G}^{M I}(s)[20,21]$. The invariant $s=f\left(\left\{p_{i} \cdot p_{j}\right\}\right)$ that is differentiated to is in general a function of the scalar products and is defined on a case by case basis.

It has been conjectured [40] that it is always possible to make a rotation of the vector $\vec{G}^{M I}(s)$ to get a DE of the following canonical form:

$$
\mathrm{DE}: \quad \frac{\partial}{\partial s} \vec{G}^{M I}(s, \varepsilon) \stackrel{I B P}{=} \varepsilon \overline{\bar{M}}(s) \cdot \vec{G}^{M I}(s, \varepsilon), \quad s=f\left(\left\{p_{i} \cdot p_{j}\right\}\right) .
$$

In this new basis the MI may be directly solved as exponentiated integrals of the matrix $\overline{\bar{M}}$. For a large class of MI these integrals are expressible in terms of GPs, which are an iterative class of functions that generalize the usual logarithms and polylogarithms [17, 19]:

$$
\begin{aligned}
& G P(\underbrace{\alpha_{1}, \cdots, \alpha_{n}}_{\text {weight } \mathrm{n}} ; x):=\int_{0}^{x} d x^{\prime} \frac{G P\left(\alpha_{2}, \cdots, \alpha_{n} ; x^{\prime}\right)}{x^{\prime}-\alpha_{1}}, \\
& G P(; x)=1, \quad G P(\underbrace{0, \cdots, 0}_{\mathrm{n} \text { times }} ; x)=\frac{1}{n !} \log ^{n}(x),
\end{aligned}
$$

where in general $\alpha_{i}, x \in \mathbb{C}$. The subsequent expansion of $\vec{G}^{M I}$ in $\varepsilon$ results in a power series whose coefficients are uniform in the weight of the GPs [40].

In the SDE approach [34] the MI are differentiated with respect to an externally introduced parameter that will be denoted by $x$. As shown on the right hand side in Figure 1, the external incoming momenta are now parametrized linearly in terms of $x$ as $p_{i}(x)=p_{i}+(1-x) q_{i}$, where the $q_{i}$ 's are a linear combination of the original external momenta $\left\{p_{i}\right\}$ such that $\sum_{i} q_{i}=0$. If $p_{i}^{2}=0$, the parameter $x$ captures the off-shellness of the massive external legs. The class of Feynman integrals in (2.1) are now dependent on $x$ through the external momenta:

$$
G_{a_{1} \cdots a_{n}}(x, s, \varepsilon)=\int\left(\prod_{i=1}^{l} d^{d} k_{i}\right) \frac{1}{D_{1}^{2 a_{1}}(k, p(x)) \cdots D_{n}^{2 a_{n}}(k, p(x))}, \quad s=\left.\left\{p_{i} \cdot p_{j}\right\}\right|_{i, j}
$$




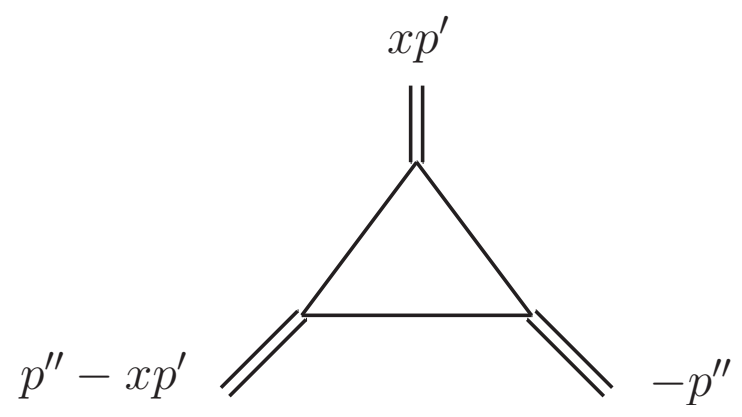

Figure 2: Required parametrization for massive triangles after possible pinchings of internal line(s).

where contrary to the traditional DE approach, the Lorentz invariants $s$ are here defined as the usual scalar products. Note that as $x \rightarrow 1$, the original configuration of the loop integrals (2.1) are reproduced. The vector $\vec{G}^{M I}(x)$ is now dependent on $x$ and one differentiates it with respect to $x$ to get a linear system of differential equations:

$$
\text { SDE: } \quad \frac{\partial}{\partial x} \vec{G}^{M I}(x, s, \varepsilon) \stackrel{I B P}{=} \overline{\bar{M}}(x, s, \varepsilon) \cdot \vec{G}^{M I}(x, s, \varepsilon), \quad s=\left.\left\{p_{i} \cdot p_{j}\right\}\right|_{i, j} .
$$

The MI with least amount of denominators $m_{0}$ are two-point integrals which can be easily calculated analytically with other methods. Furthermore, because of the form of the IBP identities (2.2) the DE of MI with $m$ denominators only depend on MI with at most $m$ denominators. This structure of the DE makes it possible to first solve the MI with $m_{0}+1$ denominators, then those with $m_{0}+2$ denominators and so forth. In other words, in practice the DE may be solved from the bottom-up. For many cases, the MI with $m_{0}$ denominators are expressible in terms of GPs (2.4) and one needs to choose the parametrization of the external momenta in $x$ such that this GP-structure for the MI with $m>m_{0}$ denominators holds as well. For the cases that we considered it was enough for us to choose the parametrization of the external legs such that after pinching internal lines the resulting triangles with three off-shell legs, if they appear, have the form given in Figure 2. In other words one of the external momentum should scale linearly with $x$, and another one should be independent of $x$.

As was noted in [34], in the SDE approach the boundary terms when solving from the bottomup are almost always naturally captured by the singularities in the SDE themselves at $x=0$, which is precisely the lower integration boundary of the GPs. In this way, the SDE method is well suited for directly and efficiently expressing the MI in terms of GPs. In particular, the boundary terms of all the two-loop planar graphs given in the next section as well as those for many other examples we have considered are captured by the SDE without the addition of any constants in $x$.

\section{Application: Master integrals of the two-loop four-point planar amplitudes with two different massive external legs}

In this section a non-trivial application of the SDE approach and partial results of [35] are presented. We are interested in calculating the MI of two-loop QCD amplitude corrections contributing to massive pair production at the LHC, where the two outgoing particles may have different masses:

$$
p p^{\prime} \rightarrow V_{1} V_{2}, \quad m_{V_{1}} \neq m_{V_{2}} \neq 0 .
$$



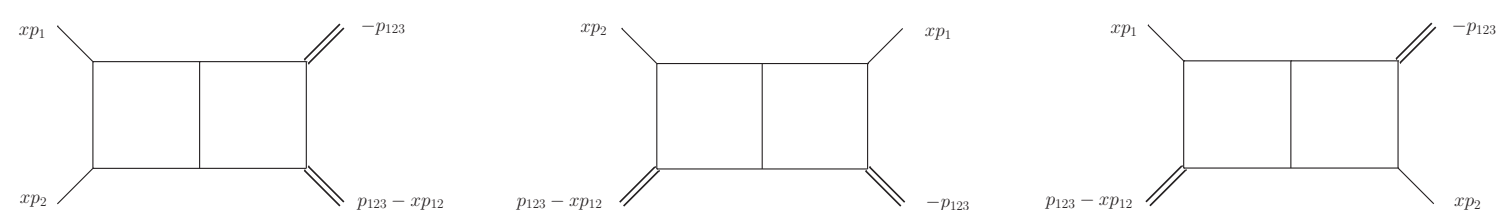

Figure 3: The parametrization of external momenta for the three planar double boxes of the families $P_{12}$ (left), $P_{13}$ (middle) and $P_{23}$ (right) contributing to pair production at the LHC.

Both the planar and non-planar diagrams have already been calculated with the traditional DE method $[32,33]$. As a comparison of similar applicability, the planar diagrams were calculated with the SDE method in [35]. These results are necessary in order to calculate two-loop QCD corrections to $W Z$ production at the LHC or in general for off-shell particle pair production.

There are three families of planar integrals whose MI with maximum amount of denominators are graphically shown in Figure 3. The three families are denoted by $P_{12}, P_{13}$ and $P_{23}$ as was done in [32] and have 31, 29 and $28 \mathrm{MI}$ respectively. In order to parametrize the external momenta we kept in mind Figure 2. In particular, the parametrization of $P_{12}$ and $P_{13}$ were chosen such as to satisfy the requirement of having massive triangles of the form shown in Figure 2 after pinching the internal line(s) between the two massless legs. The parametrization of $P_{23}$ was then found by permuting the external legs accordingly. The class of loop integrals describing the families $P_{12}$ and $P_{13}$ are explicitly expressed in $x$ as:

$$
\begin{aligned}
G_{a_{1} \cdots a_{9}}^{P_{12}}(x, s, \varepsilon) & :=\int \frac{d^{d} k_{1}}{i \pi^{d / 2}} \frac{d^{d} k_{2}}{i \pi^{d / 2}} \frac{1}{k_{1}^{2 a_{1}}\left(k_{1}+x p_{1}\right)^{2 a_{2}}\left(k_{1}+x p_{12}\right)^{2 a_{3}}\left(k_{1}+p_{123}\right)^{2 a_{4}}} \\
& \times \frac{1}{k_{2}^{2 a_{5}}\left(k_{2}-x p_{1}\right)^{2 a_{6}}\left(k_{2}-x p_{12}\right)^{2 a_{7}}\left(k_{2}-p_{123}\right)^{2 a_{8}}\left(k_{1}+k_{2}\right)^{2 a_{9}}}, \\
G_{a_{1} \cdots a_{9}}^{P_{13}}(x, s, \varepsilon) & :=\int \frac{d^{d} k_{1}}{i \pi^{d / 2}} \frac{d^{d} k_{2}}{i \pi^{d / 2}} \frac{1}{k_{1}^{2 a_{1}}\left(k_{1}+x p_{1}\right)^{2 a_{2}}\left(k_{1}+x p_{12}\right)^{2 a_{3}}\left(k_{1}+p_{123}\right)^{2 a_{4}}} \\
& \times \frac{1}{k_{2}^{2 a_{5}}\left(k_{2}-x p_{1}\right)^{2 a_{6}}\left(k_{2}-p_{12}\right)^{2 a_{7}}\left(k_{2}-p_{123}\right)^{2 a_{8}}\left(k_{1}+k_{2}\right)^{2 a_{9}}} .
\end{aligned}
$$

Similarly, the class of integrals for the family $P_{23}$ equals:

$$
\begin{aligned}
G_{a_{1} \cdots a_{9}}^{P_{23}}(x, s, \varepsilon) & :=\int \frac{d^{d} k_{1}}{i \pi^{d / 2}} \frac{d^{d} k_{2}}{i \pi^{d / 2}} \frac{1}{k_{1}^{2 a_{1}}\left(k_{1}+x p_{1}\right)^{2 a_{2}}\left(k_{1}+p_{123}-x p_{2}\right)^{2 a_{3}}\left(k_{1}+p_{123}\right)^{2 a_{4}}} \\
& \times \frac{1}{k_{2}^{2 a_{5}}\left(k_{2}-p_{1}\right)^{2 a_{6}}\left(k_{2}+x p_{2}-p_{123}\right)^{2 a_{7}}\left(k_{2}-p_{123}\right)^{2 a_{8}}\left(k_{1}+k_{2}\right)^{2 a_{9}}} .
\end{aligned}
$$

With the above parametrization, the solutions of the DE are all expressed in terms of GPs (2.4). The DE may be solved via the bottom-up approach and in this way one finds that all boundary terms are included by integrating the singularities at the boundary $x=0$ in the DE. For each family $P_{i}$, the first set of arguments $A G\left(P_{i}\right)$ appearing in the GPs, the $\alpha_{j}$ 's in (2.4), are as follows:

$$
A G\left(P_{12}\right)=\left\{0,1, \frac{m_{4}}{s_{12}}, \frac{m_{4}}{m_{4}-s_{23}}, \frac{s_{23}}{s_{12}}+1\right\}
$$




$$
\begin{aligned}
A G\left(P_{13}\right) & =A G\left(P_{12}\right) \cup\left\{\xi_{+}, \xi_{-}, \frac{m_{4}\left(m_{4}-s_{23}\right)}{m_{4}^{2}-s_{23}\left(m_{4}+s_{12}\right)}\right\}, \\
\xi_{ \pm} & =\frac{m_{4} s_{12} \pm \sqrt{m_{4} s_{12} s_{23}\left(-m_{4}+s_{12}+s_{23}\right)}}{m_{4} s_{12}-s_{12} s_{23}}, \\
A G\left(P_{23}\right) & =A G\left(P_{12}\right) \cup\left\{\frac{m_{4}-s_{23}}{s_{12}}, \frac{m_{4}}{s_{12}+s_{23}}\right\} .
\end{aligned}
$$

Note that the parameter $x$ does not appear in the first set of arguments of the GPs, but appears instead as the last argument as we integrate the DE. This can be for example explicitly seen in the solution of the scalar double box of the family $P_{13}$ :

$$
\begin{aligned}
& G_{011111011}^{P_{13}}(x, s, \varepsilon)=\frac{A_{3}(\varepsilon)}{x^{2} s_{12}\left(-m_{4}+x\left(m_{4}-s_{23}\right)\right)^{2}}\left\{\frac{-1}{2 \varepsilon^{4}}\right. \\
& +\frac{1}{\mathcal{\varepsilon}^{3}}\left[-G P\left(\frac{m_{4}}{s_{12}} ; x\right)+2 G P\left(\frac{m_{4}}{m_{4}-s_{23}} ; x\right)+2 G P(0 ; x)-G P(1 ; x)+\log \left(-s_{12}\right)+\frac{9}{4}\right] \\
& +\frac{1}{4 \varepsilon^{2}}\left[18 G P\left(\frac{m_{4}}{s_{12}} ; x\right)-36 G P\left(\frac{m_{4}}{m_{4}-s_{23}} ; x\right)-8 G P\left(0, \frac{m_{4}}{s_{12}} ; x\right)\right. \\
& \left.\quad+16 G P\left(0, \frac{m_{4}}{m_{4}-s_{23}} ; x\right)+8 G P\left(\frac{s_{23}}{s_{12}}+1, \frac{m_{4}}{m_{4}-s_{23}} ; x\right)+\cdots\right] \\
& +\frac{1}{\varepsilon}\left[9\left(G P\left(0, \frac{m_{4}}{s_{12}} ; x\right)+G P(0,1 ; x)\right)-4\left(G P\left(0,0, \frac{m_{4}}{s_{12}} ; x\right)+G P(0,0,1 ; x)\right)+\cdots\right] \\
& \left.+6\left(G P\left(0,0,1, \xi_{-} ; x\right)+G P\left(0,0,1, \xi_{+} ; x\right)\right)-2 G P\left(0,0, \frac{m_{4}}{m_{4}-s_{23}}, \frac{m_{4}\left(m_{4}-s_{23}\right)}{m_{4}^{2}-s_{23}\left(m_{4}+s_{12}\right)} ; x\right)+\cdots\right\} .
\end{aligned}
$$

The above MI is expressed in terms of a common factor $A_{3}(\varepsilon)$, the parameter $x$ and the scalar products that are related to the Mandelstam variables $S, T$ and particle masses $M_{3}, M_{4}$ as follows:

$$
\begin{array}{r}
S=s_{12} x^{2}, \quad T=m_{4}-\left(s_{12}+s_{23}\right) x, \quad M_{3}^{2}=(1-x)\left(m_{4}-s_{12} x\right), \quad M_{4}^{2}=m_{4}, \\
s_{12}=p_{12}^{2}, \quad s_{23}=p_{23}^{2}, \quad m_{4}=p_{123}^{2}, \quad A_{3}(\varepsilon)=-\frac{\Gamma(1-\varepsilon)^{3} \Gamma(1+2 \varepsilon)}{\Gamma(3-3 \varepsilon)} .
\end{array}
$$

As one may notice from the example $G_{011111011}^{P_{13}}$ above, the solutions are in general not uniform in the weight of the GPs [40]. The Goncharov polylogs can be numerically evaluated with the Ginac ${ }^{1}$ library [41]. We have not tried to simplify our analytical expressions by the use of symbol and coproduct techniques [42, 43], as we were mostly interested in showing the applicability of the SDE method, but this is expected to be possible if required. All the MI solutions of the three relevant families have been numerically compared in the Euclidean region for various phase points with the numerical program $\operatorname{Secdec}^{2}[44,45]$ and good agreement was found. For example, the numerical output of the analytical solution of the scalar double box $G_{011111011}^{P_{13}}$ at a Euclidean phase space point $x=1 / 3, s_{12}=-2, s_{23}=-5, m_{4}=-9$ equals:

\footnotetext{
${ }^{1}$ We would like to thank S. Weinzierl for his help with Ginac.

${ }^{2}$ We are thankful for the help of G. Heinrich and S. Borowka with Secdec.
} 


$$
\begin{gathered}
G_{011111011}^{P_{13}}\left(S=-2 / 9, T=-20 / 3, M_{3}^{2}=-50 / 9, M_{4}^{2}=-9\right)=-\frac{0.0191399}{\varepsilon^{4}}-\frac{0.0292887}{\varepsilon^{3}} \\
+\frac{0.0239971}{\varepsilon^{2}}+\frac{0.340233}{\varepsilon}+0.870356+\mathscr{O}(\varepsilon) .
\end{gathered}
$$

The above numerical expression has been compared with Secdec to 6 digits. For phase space points in the physical region great care needs to be taken of the Feynman $i \varepsilon_{F}$ prescription related to the propagators. We refer to [35] for the complete discussion of how our solutions may be analytically continuated to the physical region. Furthermore, the numerical comparisons at Euclidean as well as physical phase space points for all double boxes are given in [35], which will also contain all analytical solutions as ancilliary files.

\section{Conclusion and Outlook}

In this short paper we discussed an application of the SDE method proposed in [34]. Some partial results of the two-loop planar MI for two massless and two general massive external legs with massless propagators were presented. Our full results will be published in [35], where the expressions will be added as ancilliary files. All solutions are expressed in terms of GPs once the correct $x$-parametrizations of the external momenta are chosen. Furthermore, it was not needed to calculate the boundary terms as they are all captured by the singularities within the SDE method. This seems to be the case for most MI calculated with this method, which makes it an efficient and practical tool for the calculation of MI. In the future we intend to apply the method to the calculation of the corresponding non-planar MI counterparts of diboson production. No new features are expected to arise for the non-planar graphs and the SDE method should be straightforwardly applicable. In addition, the case of massive internal propagators could be the subject of future research.

\section{References}

[1] Z. Bern, L. J. Dixon, D. C. Dunbar and D. A. Kosower, Nucl. Phys. B425 (1994) 217-260, [hep-ph/9403226].

[2] Z. Bern, L. J. Dixon, D. C. Dunbar and D. A. Kosower, Nucl. Phys. B435 (1995) 59-101, [hep-ph/9409265].

[3] G. Ossola, C. G. Papadopoulos and R. Pittau, Nucl. Phys. B763 (2007) 147-169, [hep-ph/0609007].

[4] G. Ossola, C. G. Papadopoulos and R. Pittau, JHEP 0805 (2008) 004, [arXiv : 0802 . 1876 ].

[5] A. van Hameren, C. Papadopoulos and R. Pittau, JHEP 0909 (2009) 106, [arXiv : 0903.4665 ].

[6] C. Berger, Z. Bern, L. J. Dixon, F. Febres Cordero, D. Forde, et al., Phys. Rev. Lett. 102 (2009) 222001, [arXiv:0902.2760].

[7] Z. Bern, L. Dixon, F. Febres Cordero, S. Hoeche, H. Ita, et al., Phys. Rev. D88 (2013) 014025 , [arXiv: 1304.1253$]$. 
[8] A. Bredenstein, A. Denner, S. Dittmaier and S. Pozzorini, Phys. Rev. Lett. 103 (2009) 012002, [arXiv:0905.0110].

[9] G. Bevilacqua, M. Czakon, C. Papadopoulos, R. Pittau and M. Worek, JHEP 0909 (2009) 109, [arXiv:0907.4723].

[10] G. Bevilacqua, M. Czakon, C. Papadopoulos and M. Worek, Phys. Rev. Lett. 104 (2010) 162002, [arXiv:1002.4009].

[11] J. Gluza, K. Kajda and D. A. Kosower, Phys. Rev. D83 (2011) 045012, [arXiv: 1009.0472 ].

[12] D. A. Kosower and K. J. Larsen, Phys. Rev. D85 (2012) 045017, [arXiv: 1108 .1180].

[13] P. Mastrolia and G. Ossola, JHEP 1111 (2011) 014, [arXiv: 1107.6041 ].

[14] S. Badger, H. Frellesvig and Y. Zhang, JHEP 1204 (2012) 055, [arXiv: 1202 . 2019].

[15] S. Badger, H. Frellesvig and Y. Zhang, JHEP 1312 (2013) 045, [arXiv: 1310 . 1051].

[16] G. 't Hooft and M. Veltman, Nucl. Phys. B153 (1979) 365-401.

[17] A. B. Goncharov, Math Res. Letters 5 (1998) 497-516, [arXiv:1105.2076 [math. AG] ].

[18] E. Remiddi and J. A. M. Vermaseren, Int. J. Mod. Phys. A15 (2000) 725-754, [hep-ph/ 9905237$].$

[19] A. B. Goncharov, (2001) [math/0103059v4].

[20] A. Kotikov, Phys. Lett. B254 (1991) 158-164.

[21] E. Remiddi, Nuovo Cim. A110 (1997) 1435-1452. [hep-th/9711188].

[22] M. Caffo, H. Czyz, S. Laporta and E. Remiddi, Nuovo Cim. A111 (1998) 365-389, [hep-th/9805118].

[23] T. Gehrmann and E. Remiddi, Nucl. Phys. B580 (2000) 485-518, [hep-ph / 9912329 ].

[24] T. Gehrmann and E. Remiddi, Nucl. Phys. B601 (2001) 248 [hep-ph/ 0008287 ].

[25] T. Gehrmann and E. Remiddi, Nucl. Phys. B601 (2001) 287 [hep-ph/0101124].

[26] S. P. Martin, Phys. Rev. D68 (2003) 075002 [hep-ph/0307101].

[27] T. G. Birthwright, E. W. N. Glover and P. Marquard, JHEP 0409 (2004) 042 [hep-ph/ 0407343 ].

[28] M. Awramik, M. Czakon, A. Freitas and G. Weiglein, Phys. Rev. Lett. 93 (2004) 201805 [hep-ph/0407317].

[29] U. Aglietti, R. Bonciani, G. Degrassi and A. Vicini, Phys. Lett. B600 (2004) 57 [hep-ph/0407162].

[30] C. Anastasiou, S. Beerli, S. Bucherer, A. Daleo and Z. Kunszt, JHEP 0701 (2007) 082 [hep-ph/0611236].

[31] R. Bonciani and A. Ferroglia, JHEP 0811 (2008) 065 [arXiv : 0809 . 4687].

[32] J. M. Henn, K. Melnikov and V. A. Smirnov, JHEP 05 (2014) 090, [arXiv: 1402 . 70 78].

[33] F. Caola, J. M. Henn, K. Melnikov and V. A. Smirnov, [arXiv: 1404.5590 ].

[34] C. G. Papadopoulos, [arXiv:1401.6057].

[35] C. G. Papadopoulos, D. Tommasini and C. Wever, to appear on arXiv.

[36] K. Chetyrkin and F. Tkachov, Nucl. Phys. B192 (1981) 159-204. 
[37] F. Tkachov, Phys. Lett. B100 (1981) 65-68.

[38] A. von Manteuffel and C. Studerus, [arXiv:1201.4330].

[39] A. V. Smirnov, JHEP 0810 (2008) 107. [arXiv: 0807.3243 ].

[40] J. M. Henn, Phys. Rev. Lett. 110 (2013) 251601, [arXiv: 1304 . 1806].

[41] J. Vollinga and S. Weinzierl, Comput. Phys. Commun. 167 (2005) 177. [hep-ph/ 0410259 ].

[42] C. Duhr, H. Gangl and J. R. Rhodes, JHEP 1210 (2012) 075, [arXiv: 1110 . 0 458].

[43] C. Duhr, JHEP 1208 (2012) 043, [arXiv: 1203.0454$].$

[44] J. Carter and G. Heinrich, Comput. Phys. Commun. 182 (2011) 1566-1581, [arXiv: 1011.5493$].$

[45] S. Borowka, J. Carter and G. Heinrich, Comput. Phys. Commun. 184 (2013) 396-408, [arXiv:1204.4152]. 